\title{
THE ROLE OF FLEXIBLE CARBON-FIBRE IMPLANTS AS TENDON AND LIGAMENT SUBSTITUTES IN CLINICAL PRACTICE
}

\author{
A PRELIMINARY REPORT \\ D. H. R. JENKINS, B. McKIBBIN \\ From Cardiff Royal Infirmary, Cardiff
}

\begin{abstract}
The role of flexible carbon-fibre implants as substitutes for injured tendons and ligaments was investigated. These implants were simple to perform and were well tolerated by the patient. Repairs using carbon-fibres in 60 patients were successful, particularly in the almost insoluble problem of posterior cruciate laxity. The results in this report show that carbon-fibre implants have few disadvantages and have a future use in reconstructive procedures.
\end{abstract}

Previous experimental work at Cardiff Royal Infirmary has shown that implants of flexible carbonfibre may develop into satisfactory substitutes for tendons (Jenkins et al. 1977) and ligaments (Jenkins 1978). These implants are extremely well tolerated by the body in respect of the foreign body response, and their success depends on the infiltration of new collagenous tissue. The flexible carbon acts as an agent to induce the formation of a living substitute for the original structure in a unique manner (Forster et al. 1978). This has an advantage over simple prosthetic replacements, since the new implant has the power to adapt physiologically to its new role and grows stronger rather than weaker with age.

Long-term animal studies using the carbon-fibre implant have not revealed any late adverse tissue reactions, so clinical trials with the material were begun. The implantation of carbon into 102 patients and the clinical findings after one or more years in the first 60 patients are reported here. Since there is evidence that the extent of the formation of the new tendon or ligament is dependent on its anatomical environment (Ráliš and Forster, personal communication), the carbon-fibre implants were used for a wide variety of reconstructive procedures, mainly in connection with ligamentous problems around the knee.

\section{CLINICAL MATERIAL}

During the past three years flexible carbon-fibre has been implanted into 102 patients aged between 14 and 86 years. The first 60 patients have been followed up for periods of between 12 months and three years. The procedure is still in the experimental stage and therefore only those patients whose disability severely curtailed their sporting, social or working activities were selected. All the patients were operated on by the same surgeon (DHRJ).
Assessment. Thirty-six of the patients were available for reexamination and were independently assessed by the second author. The remaining 24 patients who were referred from outside Cardiff were not available for re-examination and therefore assessment was based on reports supplied by the surgeons concerned. The aim of the assessment was to grade the implant as a success or failure according to

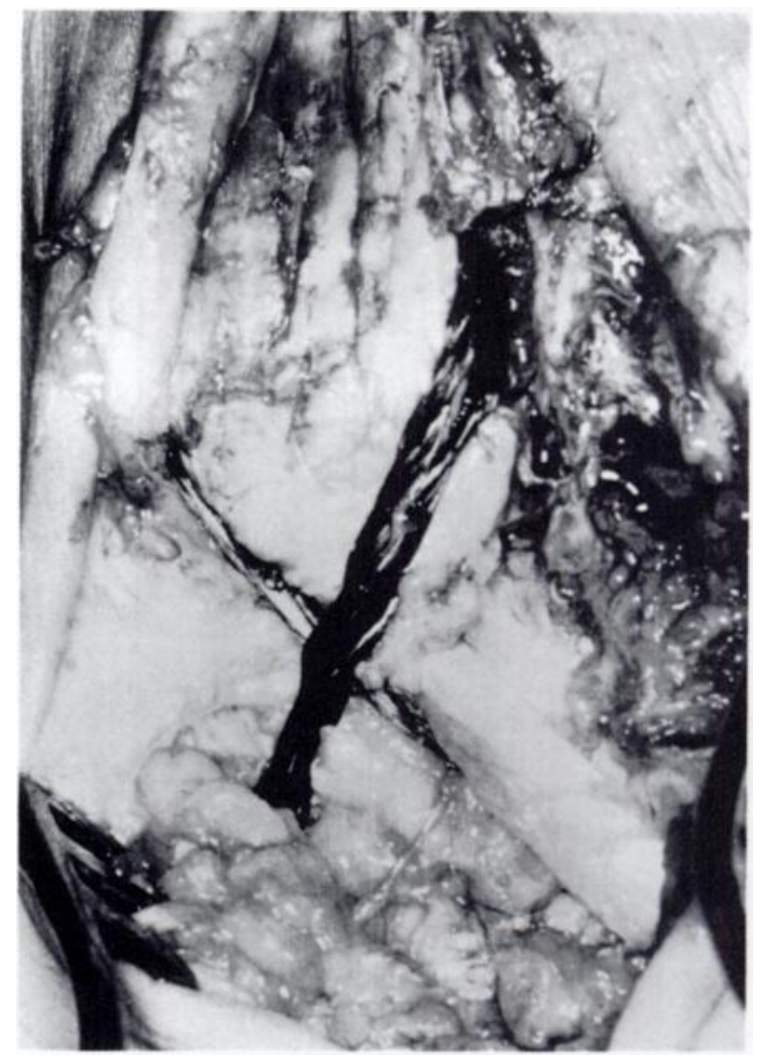

Fig. 1

Carbon-fibre spun by hand into a composite strand and used to reinforce an ankle with lateral instability.

D. H. R. Jenkins, ChM, FRCS, Consultant Orthopaedic Surgeon Cardiff Royal Infirmary, Newport Road. B. McKibbin, MD, MS, FRCS, Professor of Traumatic and Orthopaedic Surgery $\}$ Cardiff CF2 1SZ, Wales.

Requests for reprints should be sent to Mr D. H. R. Jenkins. 
both the assessor and the patient. Function before and after the operation was considered and where possible a physical examination was used to test the integrity and tension of the reconstructed ligament. The occurrence of any complications, particularly those which might indicate an adverse reaction to the material itself, were reported. Scoring system. The results were graded according to the following classification: Grade 0 -deterioration after operation; Grade 1-no change or marginal improvement; Grade 2-moderate improvement; Grade 3-significant improvement; Grade 4-restoration to complete and normal function.

The lower assessment was used whether deterioration was reported by the surgeon or the patient, since patients who know that they have had an experimental operation are often over-enthusiastic about the results.

\section{METHODS}

Quadruple strands of Courtaulds AS Grafil (10000 units per strand), which had been washed in acetone but the surface left untreated, were loosely spun by hand into a composite strand (Fig. 1) and the ends temporarily whipped with silk to prevent fraying. Normal autoclave sterilisation of the strand was possible since the material is thermostable.

Surgical technique. One continuous strand of material is usually used, even when reconstructing more than one ligament (Fig. 2). In all patients the strand was attached to the bone by passing the material through drill holes made through very limited incisions. After the carbon-fibre had passed through this hole it emerged on an appropriate and usually opposite cortical surface; it was then passed through the tissues in the line of the reconstructed ligament to a second drill hole. For the replacement of the cruciate ligaments it was necessary to open the joint (Fig. 2), and for replacements in the ankle it was found preferable to pull both ends of the carbon-fibre across the talus to emerge on the opposite side of the joint (Fig. 3). It is essential to use a drill hole of adequate diameter, since any attempt to force the carbon-fibre through a narrow space results in fraying and even disintegration of the implant.

The carbon-strand is anchored at its point of entry by a simple knot and then threaded through at the appropriate tension. The main problem is the final anchorage of the terminal strand while maintaining this tension. Our experiments with various forms of fixation have shown that simple suture to the periosteum is adequate provided the

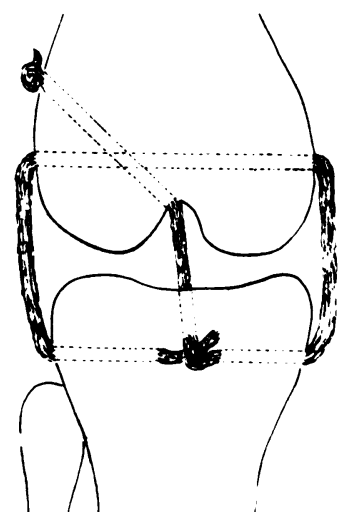

Fig. 2

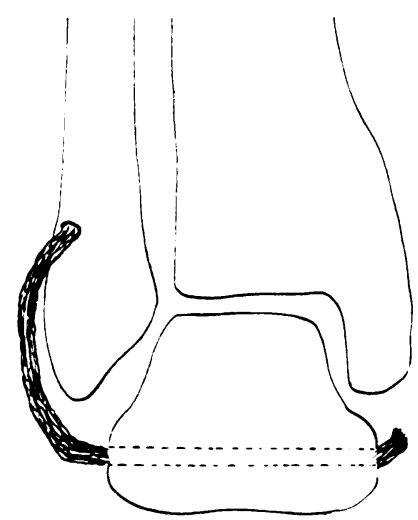

Fig. 3
Figure 2-A continuous carbon-fibre strand used in the replacement of the anterior cruciate, medial and lateral collateral ligaments of the knee. Figure 3-Repair of the lateral ligament to correct collateral ligament instability.

implant strand can be returned to its original entry point and looped through itself. The purpose of the implant is to induce the formation of a new living structure so that only temporary fixation is required. The strength of fixation is therefore much less than would be required for a normal prosthetic implant.

The repair is protected by a plaster cast for between five and six weeks to allow anchorage of the carbon-strand to take place. Forced movements are actively discouraged after removal of the plaster; after implants in the ankle, activity is restricted to walking for the first three months before running is allowed, whereas in the knee, quadriceps exercises are encouraged at this stage but not knee bending. Full sporting activities are not permitted earlier than six months after operation.

\section{RESULTS}

The results in Table I show that the majority of repairs involved the knee and ankle. In the knee the results for repairs of the collateral ligament were successful in most

Table 1 . The results of carbon-fibre implants in 60 patients

\begin{tabular}{|c|c|c|c|c|c|c|c|}
\hline \multirow[b]{2}{*}{ Repairs } & \multirow{2}{*}{$\begin{array}{c}\text { Number } \\
\text { of } \\
\text { patients }\end{array}$} & \multicolumn{5}{|c|}{ Assessment grade } & \multirow[b]{2}{*}{ Comment } \\
\hline & & 4 & 3 & 2 & 1 & $\mathbf{0}$ & \\
\hline Collateral ligaments alone (knee) & 8 & & 2 & 4 & 1 & 1 & Some loss of knee flexion. Problem in positioning \\
\hline Anterior cruciate ligaments alone (knee) & 2 & & 2 & & & & Failure to reinforce other structures \\
\hline Posterior cruciate ligaments alone (knee) & 4 & & 1 & 2 & 1 & & Major problem is positioning the carbon-fibre implant \\
\hline Combined ligamentous procedures (knee) & 31 & 10 & 7 & 13 & & 1 & A significantly useful procedure \\
\hline Medial ligament (knee) & 2 & & 2 & & & & Satisfactory procedure \\
\hline Lateral ligament (ankle) & 5 & 2 & 1 & & & 2 & $\begin{array}{l}\text { Two implants removed due to sinus formation. } \\
\text { Otherwise excellent }\end{array}$ \\
\hline Reinforcement of Freeman knee & 1 & & & & 1 & & Not useful \\
\hline Distal radio-ulnar joint & 1 & 1 & & & & & Excellent \\
\hline Shoulder capsule repair & 1 & & 1 & & & & Quite useful. Prevented arthrodesis \\
\hline Patellar tendon & 1 & & 1 & & & & Very useful procedure \\
\hline Acromioclavicular dislocation & 2 & & 1 & 1 & & & Probably not as good as the Bosworth screw \\
\hline First metacarpal joint reinforcement (thumb) & 2 & & 1 & 1 & & & Quite useful. Prevented arthrodesis \\
\hline Total & 60 & 13 & 19 & 21 & 3 & 4 & \\
\hline
\end{tabular}


cases. The exact position of the implant is important and in many of the successful cases a loss of 10 to 20 degrees of flexion was usual. Similar results were obtained with the cruciate and combined ligament repairs, although the error in placement and adjustment of tension was obviously increased. Some patients complained of a vague and indefinable discomfort within the knee. This discomfort and the loss of flexion were accepted by the patients since the implants conferred stability. The most satisfied patients were those with the greatest initial instability, even though full function was less likely to be achieved in these patients.

The results for the repair of the ankle were particularly successful, giving complete restoration of stability in all cases. One advantage of the operation was that subtalar movements were not compromised, since the repair was confined to the calcaneofibular ligaments. The other procedures were not performed in sufficient numbers to draw firm conclusions, but many of the procedures did succeed and these results can certainly be improved with more experience and the development of better techniques.

Complications. There were surprisingly few complications; in two patients with a repair of the lateral ligament of the ankle late wound breakdown and sinus formation occurred requiring removal of the implant. In both patients this was associated with a carbon-strand very close to the surface where it is not well tolerated. In a few repairs of the knee, pain and tenderness over prominent subcutaneous knots responded well to removal of the projections. Mechanical failure of the implant was a major concern, but no such failure was identified. In some of the reconstructions of the knee demonstrable instability persisted. It was suspected that this was a secondary fault caused by the physiotherapy or from a faulty positioning of the implant during the operation.

Our earlier work using animals indicated that disintegration of the filamentous carbon takes place in sheep twelve months or more after the implantation. The carbon fragments were found only in the regional lymph nodes of the sheep and not at any other site in the reticulo-endothelial system. Although biopsies of human regional nodes were not taken, no evidence of lymph node enlargement was found even after the longest follow-up.

\section{DISCUSSION}

The aim of this preliminary report has been to investigate the possible role of flexible carbon-fibre implants in the late reconstruction of ligamentous injuries. The results presented here confirm this role and justify the continued use of such implants in future repairs.

The material has been used for a wide variety of implants to determine which repair has most benefit from the carbon fibres. Many other reconstructive procedures are available, but no direct comparison has yet been made with the results for the carbon-fibre repairs. At this stage it is apparent that the carbon-fibre repairs are extremely simple to perform compared with many of the alternatives and are particularly useful in the almost insoluble problem of posterior cruciate laxity.

Apart from technical errors, especially in the early cases, the only disadvantages reported by the patients were the discomfort and the slight loss of movement in the knee. This suggests that the use of carbon-fibre implants should be confined for the present to very severe cases of disabling instability where these residual symptoms are more acceptable considering the great improvement of stability. Late mechanical failure has not been a problem with these implants so far and since the new ligament is likely to grow stronger rather than weaker with time in response to functional stress, such a problem may not occur.

Carbon-fibre implants can be obtained from Dr P. F. Lofts, Research Manager, Corporate Research and Development, Johnson and Johnson Limited, Airebank, Gargrave, Nr Skipton, Yorkshire BD23 3RX, England.

\section{REFERENCES}

Forster IW, Ráliš ZA, McKibbin B, Jenkins DHR. Biological reaction to carbon fiber implants: the formation and structure of a carbon-induced "neotendon". Clin Orthop 1978;131:299-307.

Jenkins DHR, Forster IW, McKibbin B, Ráliš ZA. Induction of tendon and ligament formation by carbon implants. J Bone Joint Surg [Br] 1977;59-B:53-7.

Jenkins DHR. The repair of cruciate ligaments with flexible carbon fibre. J Bone Joint Surg [Br] 1978;60-B:520-2. 